Paper with Special Session 8 at

IEEE European Test Symposium (ETS'16)

Amsterdam, the Netherlands - May 2016

\title{
IoT: Source of Test Challenges
}

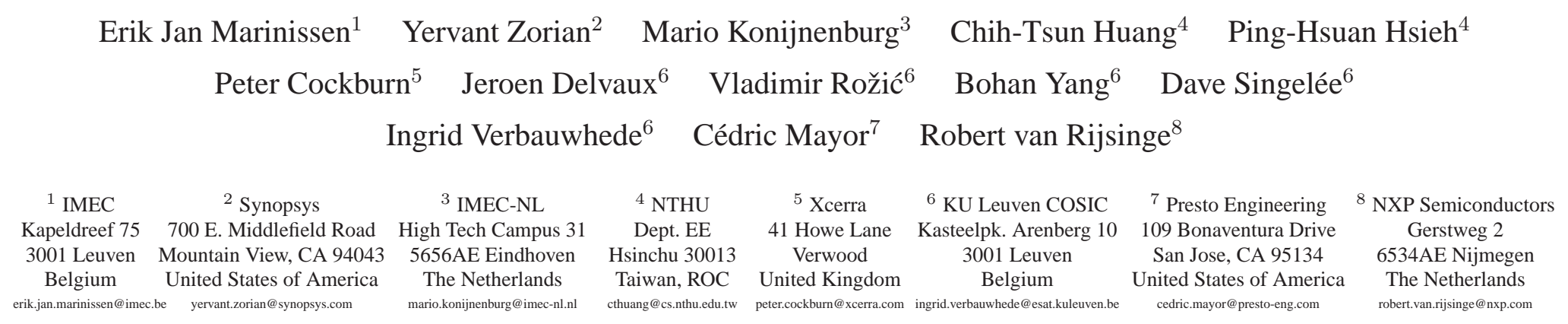

\begin{abstract}
The semiconductor industry has been driving a major part of its growth through first the PC and more recently the mobile market. Unfortunately, the PC market is in decline and also the end of the growth curve for mobile products is in sight now that virtually everyone on the planet has a smartphone and/or tablet. Hence, the semiconductor industry is putting its bets on 'Internet of Things' (IoT) as the next application wave that will allow them to sell a lot of silicon real estate. Although what exactly IoT encompasses is under definition and hence still volatile, the first emerging products depict an image which is quite different from the traditional microprocessors or smartphone SOCs: small but with ubiquitous presence, wirelessly connected, energy harvesting, equipped with smart sensors, secure, and low cost. All these aspects have a profound impact on the challenges, solutions, and associated trade-offs for testing IoT chips and provide rich grounds for research. This paper provides seven views from different angles.
\end{abstract}

\section{IoT Trends \& Test Challenges}

Yervant Zorian - Synopsys

Tbw

\section{Test Challenges in IoT from Technology and Design Point of View Mario Konijnenburg - imec-NL}

A huge amount of different kinds of devices (or nodes) with many different sensors are being connected to the internet. More than 50 billion of permanently connected devices is expected by 2020 [1]. These IoT devices interact with each other and influence in a very broad way our activities. The interaction between the devices makes the IoT system very powerful. Advanced algorithms and applications having access to a huge database of sensor data, can make very smart and independent decisions. Figure 2.1 shows a typical heterogeneous IoT network architecture with many different kinds of nodes, varying from small devices, like a temperature sensor, up to advanced devices with many sensors, and powerful routers to manage the data streams.

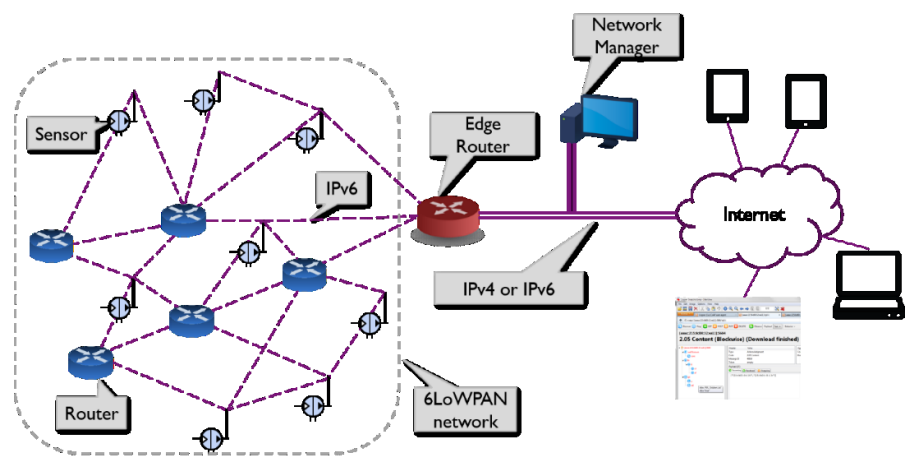

Figure 2.1: Generic IoT network architecture.

In this section a short overview is given of the design and technology aspects for IoT and their implications for tests. What kind of tests are needed? Can we afford (structural) test for ICs in the 
sensoric swarm? Is test really needed?!

\subsection{Design Architectures}

In general an IoT device consists of four parts (see Figure 2.2):

1. The sensor part. The sensor circuitry is usually analog or microelectromechanical system (MEMS) technology. The sensors can be a simple temperature sensor, an accelerometer, or an advanced ECG readout circuit [2]. In all cases the analog / MEMS part is usually significant part of the total system (chip).

2. The processing part is digital. It consists of a processor, memory, filters and accelerators for efficient processing of the sensor data streams [3]. In addition, security IP is added for secure-boot, and data encryption in memory and radio communication.

3. The radio part, which can be a Bluetooth low-energy radio, a Zigbee radio or even a Wifi radio.

4. The power management part controlling power domains, power supplies, and clock frequencies to assure efficient use of the energy (for example, by means of dynamic voltage and frequency scaling (DVFS)).

\begin{tabular}{|c|c|c|c|}
\hline ANALOG & DIGITAL & RF & \multirow{3}{*}{$\begin{array}{l}\text { Low power } \\
\text { Performance }\end{array}$} \\
\hline Sensors & $\begin{array}{l}\text { Processor } \\
\text { Memory } \\
\text { Filters } \\
\text { Accelerators } \\
\text { Security }\end{array}$ & $\begin{array}{l}\text { Radio: } \\
\text { BTLE } \\
\text { Zigbee } \\
\text { Wifi }\end{array}$ & \\
\hline \multicolumn{3}{|c|}{ Battery, Power management } & \\
\hline
\end{tabular}

\begin{tabular}{|l|l}
\hline low & performance \\
\hline deep sleep & data-rate
\end{tabular}

Figure 2.2: Generic architecture of a sensor node: consists of analog, digital, and RF with large variation in performance.

As shown in Figure 2.2, there are many different kinds of sensor nodes. Besides the purpose of the nodes and the kind of sensors, they largely can differ in processor performance, sample-rate of the data streams, on-chip memory size, the amount of activity, and the way the system is powered. All of them have influence on the way test will be performed. Small sensor nodes require a different way of test compared to large sensor nodes.
The main driver of the system development is usually the cost. Given the huge amount (billions!) of nodes, small efficient chips are needed. Analog, digital, and even RF are integrated into one die, to make the system small and low cost. Given the significant analog part, which have limited scaling benefits in new technologies, there is a tendency to continue to design in older (CMOS) technologies to stay cost efficient [4]. So what does that mean for test?

\subsection{The Test Challenge}

We can distinguish different node sizes:

- Small nodes have a cost price below one dollar-cent. Automatic structural test of these nodes would double or even triple the price. Given that these nodes are usually implemented in older technologies which have yields above $95 \%$, you may question if explicit structural test would be needed here. Placing spare sensor nodes (redundancy) might already be sufficient to have a low change in broken systems and is more cost efficient. Alternatively, applying test only on a small subset of the chips is more cost effective.

- Medium and large nodes are more expensive (ten dollarcent up to several dollars). Structural tests for these nodes makes sense. In general the yields are (a bit) lower since they are manufactured in newer and smaller technologies. However, usually large amounts of chips are needed to make the manufacturing profitable in new technologies [4], so also for these nodes old(er) technologies may be the more likely choice.

Next to structural tests, on-chip tests (built-in self-test, BIST) is an effective test method, but it is a challenge to have the full system (analog, digital and RF) properly tested by means of BIST.

\subsection{Conclusion}

Low cost is the main driver in the development of new designs and technologies for IoT. Therefore, the tendency is to stay at established (older) technologies which are less expensive and have high yields. The costs of testing these IoT design is relatively very high and might not be needed when spare sensor nodes are used in the sensoric swarm. Alternatively, BIST can be effective but is complex for designs with analog, MEMS, digital and RF. In summary, low cost and having effective tests for IoT is challenging. You may wonder if it is really needed. 


\section{IoT Test Challenges from Low Power and Energy Harvesting Viewpoints Chih-Tsun Huang, Ping-Hsuan Hsieh - National Tsing-Hua University (NTHU)}

The Internet-of-Things (IoT) has been considered as one of the emerging trends of electronics development in pervasive personal, enterprise, automotive, agriculture, life quality applications [5]. Such kinds of applications require the deployment and maintenance of massive low-power, wireless sensor nodes with minimal cost. Battery replacement is therefore infeasible during device lifetime. With severe energy limitations, these nodes tend to be self-powered with the ability of energy harvesting from their ambient environment. These IoT devices operate under low duty cycles, and are active for only a small percentage of time. Manufacturing of IoT devices is highly diversified, and so is their working environment. The diversity makes it difficult to develop a generalized design methodology and fabrication process. Effective test strategies are therefore challenging.

\subsection{Testing Energy-Harvesting Devices}

Energy sources of scavengers can be light, heat, or vibration. Recently, energy harvesting from radio frequency (RF) has also attracted increasing attention due to its non-contact, penetrative characteristics [6, 7]. Such an IoT node typically consists of a power unit, a sensing unit, a processing unit, and a communication unit, as shown in Figure 3.1 .

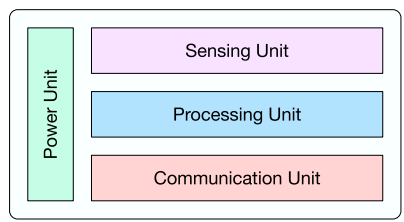

Figure 3.1: Block diagram of IoT devices.

The power unit can have two parts, the energy storage (a huge capacitor or a rechargeable battery) and the power delivery circuitry with transducer, rectifier, DC-DC conversion, and intelligent control circuit. It supplies the system either through a voltage regulator or directly. Therefore, power delivery circuitry has to be designed with the support of a wide range of current and high stability. Any design-for-test circuitry (DfT) has to be inserted carefully not to impact the power conversion efficiency. Furthermore, special equipments are needed for different energy scavengers, e.g., shakers, light sources, RF sources, etc.

The sensor unit captures different kinds of environmental information. Sensors can be either separate components, or integrated in the same package. Testing these MEMS-based devices relies a different domain knowledge.

Finally, the processing unit may consist of digital cores such as processors, accelerators, and memory. The communication unit includes analog, mixed-signal (AMS) and RF blocks for wireless access to the outside world. Power-aware test strategies should be carefully applied to test both of them.

\subsection{Power-Aware Test Strategies}

Testing IoT devices of stringent power and energy limitation could be a big burden [8]. These massive devices are usually cost-sensitive. However, the need of test-cost reduction conflicts with test power reduction, because test with higher efficiency implies a higher toggle rate. In addition, testing several modules in parallel has become a common practice to shorten the test time. Both will lead to excessive power consumption during testing, resulting in yield loss or reliability issues due to the overstress. The test power problems will become even more serious when the testing is applied at speed to ensure test quality. In addition, the DfT circuitry or built-in self test (BIST) circuitry may also consume power in function mode, decreasing the system uptime of the battery-powered devices.

Therefore, power-aware test will be a major consideration for manufacturing of IoT devices. Several techniques, which have also been investigated in recent years, include power-aware test pattern generation, hierarchical test with power management, low-power logic/memory BIST, and leakage test power reduction, etc.

Testing the power management itself is also challenging. The complicated power modes may involve sophisticated control of multiple power supplies and domains, dynamic voltage/frequency scaling, clock isolation circuitry, and power isolation circuitry. Functional test is more efficient than repeated test over all possible modes. In addition, test power can stay within the limitation. This can be achieved by developing subtle self-test capability with low-cost testers.

Test of AMS and RF modules is usually ad hoc and design specific, which means the test is commonly functional. However, self-test of these devices is usually not straightforward with a lot of engineering effort.

\subsection{Reliability Issues}

With the exposure to the outside environment, reliability of IoT devices can be a critical issue, especially with low-cost packages. Aging and environment variation (such as temperature changes) can even cause drifts of load current from the power delivery circuitry. Low-cost packaging adds variations and further increases the load current ranges. Hence self-test, self-diagnostic, and selfrepair has to be utilized at system level.

On-line testing introduces another challenge. Not only the system power level is constrained in the field, but the available energy cannot be guaranteed all the time. The energy available for 
in-field test is limited by the storage condition. Energy-aware or scalable test should be considered to be able to change the test flow adaptively based on different test conditions. To maximize the efficiency, in-field test needs to be performed in a distributed manner among the sensor network.

In-field self-repair will bring the challenge to another dimension.
Approximate computing or error resilience may be an alternative solution when coordinating both hardware and software components in the system. There are challenges and opportunities to bring the brilliant IoT vision into reality. Multi-discipline coordination is a must to develop design, manufacturing, and test processes of IoT devices.

\section{Testing Challenges for IoT Smart Sensors \\ Peter Cockburn - Xcerra Corporation}

According to many market predictions, the Internet of Things will drive a 'third wave' of semiconductor growth to enable over 25 billion sensors by 2020 for end-node applications in multiple market segments [9]. These IoT end-nodes are smart, connected 'things' that typically consist of three key elements: sensors, microcontrollers (including flash memory), and low-power wireless interfaces; see Figure 4.1. This functional integration, combined with the need to perform final test and calibration of different kinds of sensor nodes, is creating a multi-dimensional test challenge in manufacturing. This section reviews aspects of the different test challenges and proposes solutions to answer them in a cost-effective way.

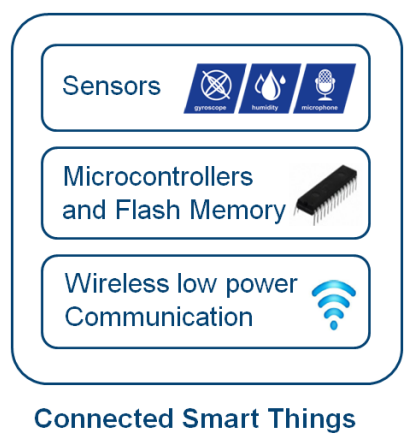

Figure 4.1: IoT end-nodes: 'connected smart things'.

\subsection{IoT Sensor Trends}

The IoT is the network of physical objects that contain embedded technology to communicate and sense or interact with their internal states or the external environment [10].

In recent years, many sensor types migrated from larger, analog architectures into semiconductor (MEMS) implementations, enabling several factors of miniaturization and resulting cost reduction. This high level of sensor integration has supported the smartphone revolution, delivered new levels of automotive performance, and enabled many other new applications. A general challenge for sensor manufacturing is the requirement to calibrate the sensor at the end of the manufacturing process, prior to shipment. This requires a non-electrical stimulation (e.g., grav- ity, pressure, light, magnetic field) applied to the sensor during electrical final test.

Microcontrollers in IoT end-nodes are SOCs in their own right, with embedded memory, mixed-signal functions and security features that require more complex test capabilities.

Numerous RF protocols and interfaces are being proposed for various industrial, personal, and domestic IoT applications, requiring a flexible and low-cost RF test solution. It can be seen that the connection of the sensors via RF communication links and the integration of data processing using an integrated micro controller is further increasing the complexity and diversity of manufacturing test requirements.

\subsection{IoT Test and Manufacturing Challenges}

A universal challenge is the cost pressures driven by falling average sales prices (ASPs) for sensors, microcontrollers, and wireless ICs. Low-cost test solutions are required, but new technologies and applications are making it difficult to define and stick to a cost-effective IoT test standard:

- Innovative and smaller packaging (WLCSP, SiP, 3D, Embedded) with finer contact pitches, must be handled reliably with lowest conversion efforts and costs.

- New sensor applications require significant test setup adaptations: every kind of sensor (e.g., accelerometer, gyroscope, magnetometer, pressure, humidity, light, IR) requires a new stimulus module.

- Diverse market segments (consumer, automotive, industrial, medical) are demanding solutions at different specifications and conditions (ambient vs. tri-temp).

- Continuous ASP reduction drives a need for higher parallelism to achieve acceptable cost of test, which in turn mandates tester architectures with high multi-site efficiency.

Other challenges come from the higher levels of integration. 
- Multiple sensors integrated in one package will require different types of stimulus and this could become a cost-oftest roadblock, unless multiple stimuli can be combined in one system.

- The integration of low-power microcontroller cores, memory, RF, sensors and smart power leads to contradictory test scaling requirements and capabilities, where the boundaries might come from the tester instrumentation or the test handler interfaces.

IoT solutions are targeting a broad mix of applications, many of which will drive high volumes with short product life-cycles. Dynamic market requirements may include new wireless protocols, new sensor functions and protocol-based programming. U1timately this will lead to ramp requirements that exceed the slope of today's mobility product ramps.

\subsection{Solutions for IoT End-Node Test}

In order to address the test and manufacturing challenges of IoT products, we have defined three key characteristics for an IoT end-node test cell (cfr. Figure 4.2): (1) low cost-of-test, (2) flexible to meet today's challenges, and (3) adaptable to future needs. In addition the test cell needs to support the different test requirements of all the end-node components: specific sen- sor stimulus, power management technology, low-cost RF covering all wireless protocol standards, and digital protocol engines. All of these test requirements need to implemented in-line with high-volume production requirements: multi-package handling technology, high multi-site testing capability, robust contacting, performance-optimized, flexible test cell. Even though these are general requirements, it is mandatory to support them at all component levels of the test cell: the tester, the test handler, the stimulus module, and the interface products. Typically this can only be managed with a holistic approach that provides a common point of ownership for the key test cell components.

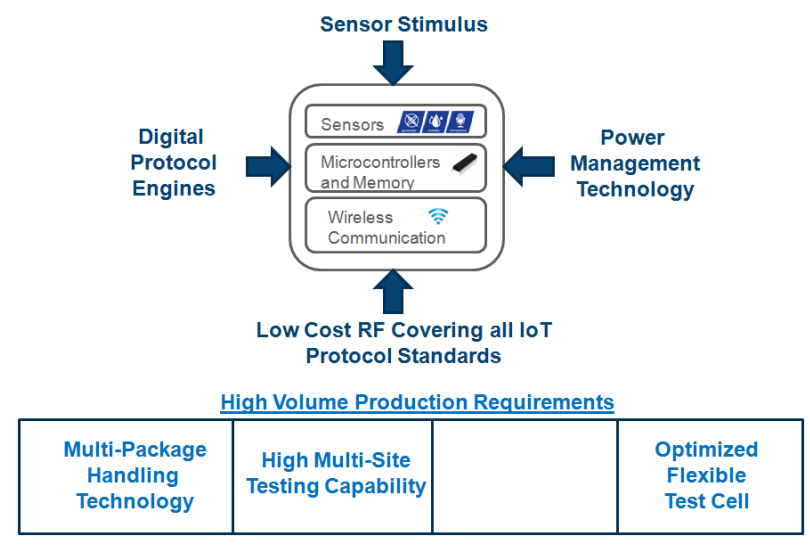

Figure 4.2: Key capabilities for IoT test cell.

\section{Testing of Random Numbers and PUFs: Roots of Trust for IoT}

Jeroen Delvaux, Vladimir Rožić, Bohan Yang, Dave Singelée, Ingrid Verbauwhede - KU Leuven - COSIC

The security architecture of any computing device is typically built with multiple levels of abstraction, where each layer trusts the layers below. The core of this system is the so-called 'root of trust', a set of functions trusted by all other components in the device. These functions are mostly embedded in hardware, since solutions that are only based on software can be bypassed by low-level attacks. Two hardware roots of trust, particularly interesting in the context of IoT, are Physically Unclonable Functions (PUF) and True Random Number Generators (TRNG). At the highest abstraction layers, PUFs are functions that produce a binary output when given a binary input, while TRNGs are functions that produce a binary output (not based on any external input).

Cryptography relies on reproducible uniformly distributed secret keys. Obtaining affordable physically secure key-storage in embedded non-volatile memory (NVM) is hard though. Harvesting entropy from PUFs comprehends an alternative that lowers the power-off state vulnerability. Likewise, cryptographic algorithms, protocols and masking-based countermeasures against physical attacks rely on high-entropy random numbers. TRNGs are building blocks to generate random numbers from a physical stochastic process, such as clock jitter. Unfortunately, the raw output of both PUFs and TRNGs could contain weaknesses or failures. High-quality, lightweight testing is therefore required.

It is important to note the the nature of testing for roots of trust is different than other domains. The normal definition of testing refers to digital tests for manufacturing defects, such as bridge faults, stuck-at faults, etc. However, this testing definition does not apply in the context of roots of trust. The key problem is that PUFs and TRNGs are non-digital components, and therefore digital tests are not sufficient. Even more, also testing at run-time is required, while being deployed in the field, since PUF or TRNG failures could be caused by changing the environmental conditions or due to manipulations of an adversary.

\subsection{Test Requirements}

The test requirements for PUFs and TRNGs are not the same. For example, noise in the PUF output is an undesired property and is mitigated by applying helper data algorithms. However, in TRNGs, noise that originates from the physical stochastic processes is required to create irreproducible randomness. Similarly, variability is an important property in PUFs, but not in TRNGs. 
The uniformity of both PUF and TRNG outputs needs to be tested. The NIST test suite [11] is frequently used to detect nonuniformities in TRNGs, but should be adopted with care when applied to PUFs. The fact that its statistical tests operate on one-dimensional (1D) bitstreams is convenient for TRNGs, but highly problematic for PUFs. It is an occasional practice [12, 13] to feed PUF outputs directly into the test suite, while at least in part ignoring the inputs. Unfortunately, non-uniformities that manifest as input-output correlations are easily overlooked then, as illustrated in the two examples below.

A prominent category of PUFs consists of a two-dimensional (2D) array of identically designed cells, each producing a single output bit. This includes, for example, the SRAM PUF [14] as well as some ring oscillator-based designs [15]. Inputs that address the array mark cells for read-out. When reordering the 2D array contents into a 1D stream, spatial correlations among neighboring cells are clearly hidden. We argue that a more suitable battery of statistical tests should be developed, explicitly incorporating the $2 \mathrm{D}$ structure.

The issue is even amplified for a second category of PUFs, producing highly correlated input-output pairs due to functional dependencies. This includes the arbiter PUF [16]. Given a relatively small training set of input-output pairs, an accurate predictive model of the PUF can be obtained via machine learning [17]. Uniformity tests that ignore the inputs are hence clearly inadequate.

For TRNGs, applying the NIST test suite [11] to assess statistical properties is necessary but not sufficient to guarantee uniformity of the output bits. Several implementations of statistical tests have been presented in the past, e.g., [18-21]. However, these tests are not suitable for assessing raw random numbers because they are designed for an ideal randomness source. A formal security evaluation, using a stochastic model of the process that generates the randomness, is required to quantify the amount of generated entropy.

The output bits of the TRNG should be perfectly random, as these bits are used for generating keys, challenges, masks and secret shares. Even when strong cryptography is used, the security of the system will be compromised when an insecure TRNG is used to generate the cryptographic keys. For example in 2012, two research studies [22, 23] found thousands of insecure RSA moduli. The problem was traced back to a fault in a random number generator. A follow-up research [24] found compromised RSA keys from Taiwan's national Citizen Digital Certificate database. Again, the problem was caused by faulty RNGs embedded in smart cards, which produced predictable numbers.

\subsection{Required Building Blocks}

Both PUFs and TRNGs need to be assisted by additional building blocks. PUF outputs are corrupted by noise and non-uniformities are bound to occur. Various helper data algorithms [25] are used to convert the raw PUF outputs into stable high-entropy keys. This includes error handling and the compression of the output bits of the PUF to a shorter string of high-entropy bits. The helper-data that is used in the error handling can be stored in read-insecure non-volatile memory as the protocol construction is such that revealing the helper data does not reveal the key. Protecting its integrity is recommended though given that manipulation attacks could reveal the key. On top, a cryptographic check can confirm whether the key has been reconstructed correctly. This deterministic validation at run-time differs from TRNGs, where different testing components are needed.

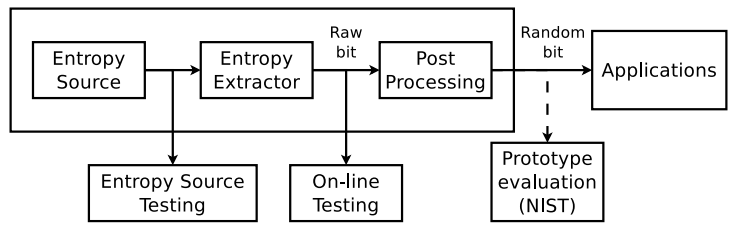

Figure 5.1: Generic test architecture of a true random number generator.

Figure 5.1 shows the generic test architecture of a TRNG. The entropy source is the component that produces randomness, for example a free running ring oscillator or a noisy resistor. The entropy extractor converts this randomness into digital numbers. Unfortunately, this raw output is usually not statistically perfect. Bias (the imbalance in the number of zeros and ones) and dependencies between the generated bits exist and post-processing is required to convert them into a usable full-entropy bit string. Post-processing used in TRNGs compresses the string of raw random numbers into a shorter string of perfectly random bits. According to NIST SP800-90B [26] and AIS31 [27] recommendations, online testing should be applied on the raw random numbers because statistical defects caused by faults and attacks can be masked by the post-processing algorithm, making the attack detection more difficult. An experiment-oriented test design methodology called TOTAL (TRNG On-the-fly Testing for Attack detection using Lightweight hardware) was presented in [28]. This methodology is based on observing the effects of low-cost attacks on the statistical features of the generated raw numbers, and finding an optimal feature for test design. This methodology results in lightweight online tests, tailored for a given entropy source, with high attack-detection capabilities.

\subsection{Conclusion}

PUFs and TRNGs are used as hardware roots of trust in IoT devices, as they fulfill the essential need of generating secure keys and nonces respectively. However, lightweight high quality testing of TRNGs and PUFs is absolutely required to exploit the full potential of these building blocks. Without these tests, non-intentional failures or dedicated attacks are not detected, and hence pose significant security hazards to IoT devices. The design and implementation of lightweight but nevertheless highquality testing methods is an active open research problem.

\section{Acknowledgements}

This work was supported in part by European Union's Horizon 2020 research and innovation programme under grant agreement No 644052 HECTOR. 


\section{Route of Trust for IoT Manufacturing Test \\ Cédric Mayor - Presto Engineering Inc.}

\subsection{Impact on the SiP KGD Test Flow}

First IoT products today are mainly SiPs grouping MCU, RF, and sensor ICs in a single package. SiP IC packaging is in itself not a new concept, but from a manufacturing-test point of view it is, and can be very demanding in test coverage requirements to deliver acceptable cost and quality trade-off. A known-good die (KGD) test flow uses three independent test routes, and commonly three different ATE machine types to match and check the specific features of each active IC component. MEMS and RF dies require specific handling/physical-stimulus fixtures and RF instruments addressed via dedicated measurement pins. The left side test flow in Figure 6.1 illustrates how we could accommodate the heterogeneous nature of such an IoT SiP by re-using today's SiP-KGD test approach and map what might be considered unique onto an industry-standard business practice.

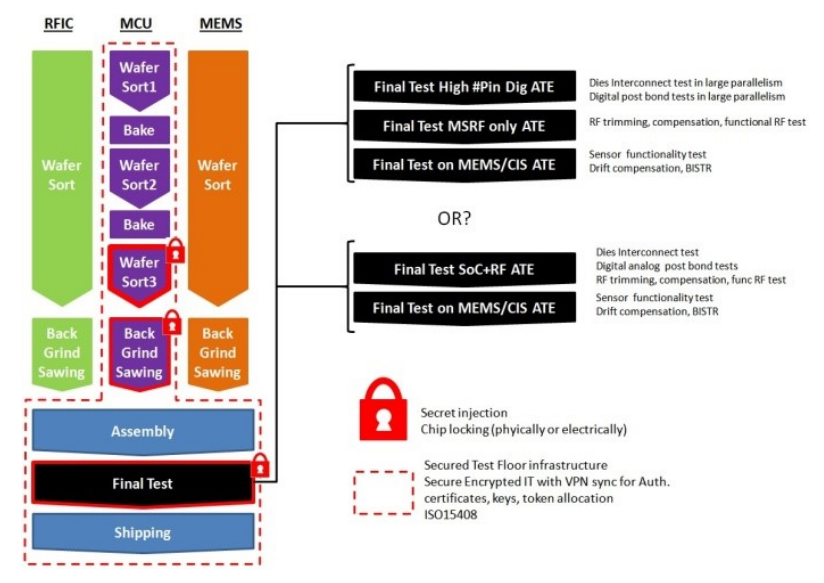

Figure 6.1: KGD test flow example for IoT devices.

In general the test flow requires for each bare die to be fully tested BIN1, and then so refered to as KGD. From a cost standpoint, it means that each tested die then already carries the cost of non quality adder of front-end baseline defects and test-related yield losses. Then the assembly process is either based on traditional SiP technology such as wire-bonding (stacked die or side-byside), reconstituted wafer-level packages, or more recently the 3D-SiP stacking with a through-silicon vias (TSVs), and redistribution layer (RDL) with micro-bumping.

These mainstream back-end and packaging processes, or the one currently being adopted by the market, can generate specific random defect types. For instance, wafer thinning and back-lap generate bare-die-level defects such as interface delamination, most of the time resulting in cracks/voiding migration, but also die surface damages, contamination or scratches, thin film or pas- sivation cracking, or die chipping. In addition some macro defects are typically dependent of the pure bonding process and can be detected by in line optical and $\mathrm{x}$-ray inspection like wire lifted/broken wires/bonds, wire bond pads peel-off, bump voids, mis-alignment, bump cracks/shearing. Smaller size defects can only be captured by an appropriate final test at package level.

Thus a SiP final test process initial focus is to first test the chip interconnects. Standardization of the test access mode since now the advent of IEEE Std 1149.1 [29] makes this interconnect structural test sanity check possible with very limited overhead in test time and full re-use of die DfT. The standard is still being amended to cover even more complex packaging and stacking processes such as TSV with the IEEE Std P1838 recently initiated [30].

Defects covered by such interconnect tests are typically modeled with usual fault models, such as stuck-at, bridging, and delay fault; however, they cannot cover for parametric yield losses. Parametric yield losses are very dependent on the SiP interconnect quality impacted by wire self/mutual-inductance, parasitic capacitance bump/RDL to substrate for RF, local self-heating, or even resistivity of connections. In essence AC analog signals are highly dependent on these physical parameters, and therefore the final test has also to cover for the induced faults. Built-in selftest and self-healing techniques are thus seen as two key pillars of IoT SiP testability, given that internal signals are often inaccessible by standard external ATE. These are proven techniques in single package test notably with MEMS products, where the companion ASIC is consistently compensating the imperfections and drifts of the sensor response by self calibration, and in the memory products where BIST is widely used to lower test time, fan-out, and repair potential defectives arrays/sectors. However RF devices do not typically contain BIST approaches in contrast with state-of-the-art SiP testing [31]. Most RF transceivers rely on the modem baseband interface to calibrate and compensate for RF performance variations. Therefore an IoT SiP will need a final test on an ATE which might handle both physical stimuli with its appropriate fixture and RF source/measurement capabilities. Coming back to Figure 6.1, such ATE systems do not exist today, and thus any final test will need to be performed in sequence on different ATEs, which will add to the total cost.

\subsection{Impact of Security on the IoT SiP Test Flow}

The first and foremost concern about IoT product adoption is security. Lack of security is often at the top of the list of concerns when talking about an autonomous object connected to a network. Connected objects with either a backdoor or counterfeit 
devices inside a SiP could jeopardize the whole system infrastructure of a service provider.

The semiconductor industry has already encountered such problems with test manufacturing flows for the smartcards and other secured products. These experiences have had a strong impact on the manufacturing test infrastructure and complexity of testing. To connect to a network autonomously and safely, a device need to use an encrypted certificate authentication approach, or a process based on secret token keys, to insure unique signatures. Therefore the IoT die and SiP module must contain a certain level of confidentiality, and feature a lock-down secret code zone. The big challenges become then threefold.

- How to ensure each die in an IoT SiP/MCM device can be tested along a route of trust similar to secure smart card approaches?

- How can the test service provider guarantee a seamless manufacturing test while at the same time injecting secrets from customers, without storing the secret information, needing to decrypt it, leaving it open to reverse engineering, while also detecting any manufacturing quality issues?
- How does injecting secrets/certificates impact the DfT, and diagnosis of the chip?

Inserting secret keys, tokens, certificates, and boot loaders into the device during wafer probe or ultimately at final test on package level is a sensitive operation. The secrets have to be dynamically allocated to the die and are often reshuffled by the endcustomers to disaggregate the supply chain. This places significant demands and requires that the test floor be equipped with encrypted server gateways, with the ability of pushing selectively the encrypted information into the right device on wafers, which most of the time is locked at the end of the wafer test and completely isolated when the wafer is sawn. Figure 6.1 represents a typical security shell that has to be in place to protect the sensitive die, and at test insertion where encrypted secrets programming/verification has to be enabled.

This is essential since an IoT device is heterogeneous by nature and will demand sourcing dies from different vendors with the need to validate the trust of each component. Recent examples in the industry showed that software is an important part of the trust chain and security channel, but hardware is the foundation of paving such a route of trust to be able to build on a solid ground.

\section{Achieving $100 \times$ Test Cost Reduction: a Dream or Reality? \\ Robert van Rijsinge - NXP Semiconductors}

Tbw

\section{References}

[1] Dave Evans. The Internet of Things - How the Next Evolution of The Internet is Changing Everything. Cisco Internet Business Solutions Group (IBSG), April 2011. white paper: http://www.cisco.com/c/dam/en_us/about/ac79/docs/innov/IoT_IBSG_ 0411FINAL.pdf.

[2] Nick Van Helleputte et al. A Multi-Parameter Signal Acquisition SoC for Connected Personal Health Applications. In Proceedings International Solid State Circuits Conference (ISSCC), pages 314-315, 2014. doi 10.1109/ISSCC.2014.6757449

[3] Mario Konijnenburg et al. A Battery-Powered Efficient Multi-Sensor Acquisition System with Simultaneous ECG, BIO-Z, GSR and PPG. In Proceedings International Solid State Circuits Conference (ISSCC), pages 480-481, 2016.

[4] Michael White. IoT, Cost-per-Transistor Extend Lifetimes of Established Technology Nodes. Electronic Design, May 2015. http://electronicdesign.com/eda/iot-cost-transistor-extend-lifetimesestablished-technology-nodes.

[5] Sandip Ray, Yier Jin, and Arijit Raychowdhury. The Changing Computing Paradigm With Internet of Things: A Tutorial Introduction. IEEE Design \& Test, 33(2):76-96, April 2016. doi 10.1109/MDAT.2016.2526612

[6] Ruud J.M. Vullers and Rob van Schaijk and Hubregt J. Visser and Julien Penders and Chris Van Hoof. Energy Harvesting for Autonomous Wireless Sensor Networks. IEEE Solid-State Circuits Magazine, 2(2):29-38, Spring 2010. doi 10.1109/MSSC.2010.936667
[7] Ping-Hsuan Hsieh, Chih-Hsien Chou, and Tao Chiang. An RF Energy Harvester With 44.1\% PCE at Input Available Power of -12 dBm. IEEE Transactions on Circuits and Systems - Part I: Regular Papers, 62(6):1528-1537, June 2015. doi 10.1109/TCSI.2015.2418834

[8] C.P. Ravikumar, Moktar Hirech, and Xiaoqing Wen. Test Strategies for Low Power Devices. In Proceedings Design, Automation, and Test in Europe (DATE), pages 728-733, March 2008. doi 10.1109/DATE.2008.4484765

[9] Rob Lineback, Bill McClean, and Brian Matas. Integrated Circuit Market Drivers 2016. IC Insights, Inc., November 2015.

[10] Gartner. On-Line IT Glossary, 2016. http://www.gartner.com/itglossary/internet-of-things.

[11] Andrew Rukhin et al. A Statistical Test Suite for Random and Pseudorandom Number Generators for Cryptographic Applications. NIST Special Publication 800-22, 2010. http://csrc.nist.gov/publications/nistpubs/80022-rev1a/SP800-22rev1a.pdf.

[12] Cheng Wei Lin and Swaroop Ghosh. A Family of Schmitt-Trigger-Based Arbiter-PUFs and Selective Challenge-Pruning for Robustness and Quality. In IEEE International Symposium on Hardware Oriented Security and Trust (HOST), pages 32-37, May 2015. doi 10.1109/HST.2015.7140232

[13] Chi-En Daniel Yin and Gang Qu. Obtaining Statistically Random Information From Silicon Physical Unclonable Functions. IEEE Transactions on Emerging Topics in Computing, 2(2):96-106, 2014. doi 10.1109/TETC.2014.2316497

[14] Daniel E. Holcomb, Wayne P. Burleson, and Kevin Fu. Power-Up SRAM State as an Identifying Fingerprint and Source of True Random 
Numbers. IEEE Transactions on Computers, 58(9):1198-1210, 2009. doi 10.1109/TC.2008.212

[15] Haile Yu et al. Towards a Unique FPGA-Based Identification Circuit Using Process Variations. In International Conference on Field Programmable Logic and Applications (FPL), pages 397-402, 2009. doi 10.1109/FPL.2009.5272255

[16] Daihyun Lim et al. Extracting Secret Keys from Integrated Circuits. IEEE Transactions on VLSI Systems, 13(10):1200-1205, 2005. doi 10.1109/TVLSI.2005.859470

[17] Ulrich Rührmair et al. PUF Modeling Attacks on Simulated and Silicon Data. IEEE Transactions on Information Forensics and Security, 8(11):1876-1891, 2013. doi 10.1109/TIFS.2013.2279798

[18] Renaud Santoro, Olivier Sentieys, and Sébastien Roy. On-line Monitoring of Random Number Generators for Embedded Security. In Proceedings International Symposium on Circuits and Systems (ISCAS), pages 30503053, 2009. doi $10.1109 /$ ISCAS.2009.5118446

[19] Filip Veljkovic, Vladimir Rozic, and Ingrid Verbauwhede. Low-Cost Implementations of On-the-Fly Tests for Random Number Generators. In Proceedings Design, Automation, and Test in Europe (DATE), pages 959-964, 2012. doi 10.1109/DATE.2012.6176635

[20] V.B. Suresh, D. Antonioli, and W.P. Burleson. On-Chip Lightweight Implementation of Reduced NIST Randomness Test Suite. In IEEE International Symposium on Hardware Oriented Security and Trust (HOST), pages 9398, 2013. doi 10.1109/HST.2013.6581572

[21] Bohan Yang et al. Embedded HW/SW Platform for On-the-Fly Testing of True Random Number Generators. In Proceedings Design, Automation, and Test in Europe (DATE), pages 345-350, March 2015.

[22] Arjen K. Lenstra et al. Public Keys. In Advances in Cryptology (CRYPTO), pages 626-642, 2012. doi 10.1007/978-3-642-32009-5_37

[23] Nadia Heninger et al. Mining Your Ps and Qs: Detection of Widespread Weak Keys in Network Devices. In Proceed- ings of the 21th USENIX Security Symposium, pages 205-220, 2012. https://www.usenix.org/conference/usenixsecurity12/technicalsessions/presentation/heninger.

[24] Daniel J. Bernstein et al. Factoring RSA Keys from Certified Smart Cards: Coppersmith in the Wild. In Advances in Cryptology (ASIACRYPT), pages 341-360, 2013. doi 10.1007/978-3-642-42045-0_18

[25] Jeroen Delvaux et al. Helper Data Algorithms for PUF-Based Key Generation: Overview and Analysis. IEEE Transactions on Computer-Aided Design, 34(6):889-902, 2015. doi 10.1109/TCAD.2014.2370531

[26] Elaine Barker and John Kelsey. Recommendation for the Entropy Sources Used for Random Bit Generation. NIST DRAFT Special Publication 800-90B, 2012. http://csrc.nist.gov/publications/drafts/800-90/draftsp800-90b.pdf.

[27] Wolfgang Killmann and Werner Schindler. A Proposal for: Functionality Classes for Random Number Generators. BDI, Bonn, 2011. https://www.bsi.bund.de/SharedDocs/Downloads/DE/BSI/Zertifizierung/ Interpretationen/AIS_31_Functionality_classes_for_random_number_ generators_e.pdf?__blob=publicationFile.

[28] Bohan Yang et al. TOTAL: TRNG On-the-Fly Testing for Attack Detection using Lightweight Hardware. In Proceedings Design, Automation, and Test in Europe (DATE), March 2016.

[29] IEEE Standards Association. IEEE Std 1149.1 TM -2013 , IEEE Standard for Test Access Port and Boundary-Scan Architecture. IEEE, New York, NY, USA, May 2013. doi 10.1109/IEEESTD.2013.6515989

[30] Erik Jan Marinissen, Teresa McLaurin, and Hailong Jiao. IEEE Std P1838: DfT Standard-under-Development for 2.5D-, 3D-, and 5.5D-SICs. In Proceedings IEEE European Test Symposium (ETS), 2016.

[31] Yervant Zorian and Hakim Bederr. An Effective Multi-Chip BIST Scheme. Journal of Electronic Testing: Theory and Applications, 10(1):87-95, February 1997. doi 10.1023/A:1008226715929 